\title{
Multiple sclerosis and depressive manifestations: can diffusion tensor MR imaging help in the detection of microstructural white matter changes?
}

\author{
Talaat A. Hassan', Shaima Fattouh Elkholy ${ }^{1}$, Bahaa Eldin Mahmoud ${ }^{1 *}$ (ID and Mona ElSherbiny ${ }^{2}$
}

\begin{abstract}
Background: Multiple sclerosis is one of the commonest causes of neurological disability in middle-aged and young adults. Depression in MS patients can compromise cognitive functions, lead to suicide attempts, impair relationships and reduce compliance with disease-modifying treatments. The aim of this study was to investigate and compare the microstructural changes in the white matter tracts of the limbic system in MS patients with and those without depressive manifestations using a diffusion tensor imaging (DTI) technique.

Methods: This study included 40 patients who were divided into three groups. Group 1 comprised of 20 patients with relapsing-remitting MS with depressive symptoms and group 2 comprised 10 MS patients without symptoms of depression. The third group is a control group that included 10 age-matched healthy individuals. All patients underwent conventional MRI examinations and DTI to compare the fractional anisotropy (FA) values in the white matter tracts of the limbic system.

Results: We compared the DTI findings in MS patients with and those without depressive symptoms. It was found that patients with depression and MS exhibited a significant reduction in the FA values of the cingulum $(P<0.0111$ on the right and $P<0.0142$ on the left), uncinate fasciculus ( $P<0.0001$ on the right and $P<0.0076$ on the left) and the fornix $(P<0.0001$ on both sides). No significant difference was found between the FA values of the anterior thalamic radiations in both groups.

Conclusion: Patients with depression and MS showed more pronounced microstructural damage in the major white matter connections of the limbic pathway, namely, the uncinate fasciculus, cingulum and fornix. These changes can be detected by DTI as decreased FA values in depressed MS patients compared to those in non-depressed patients.
\end{abstract}

Keywords: Diffusion tensor imaging, Limbic system, Depression, Multiple sclerosis

\section{Background}

Multiple sclerosis is one of the commonest causes of neurological disability in middle-aged and young adults. Demyelination, inflammation and axonal damage are the pathological hallmarks giving rise to the characteristic multifocal CNS lesions seen in MS particularly targeting the periventricular, juxta cortical regions, brainstem, optic nerves and spinal cord [1].

\footnotetext{
* Correspondence: Bahaa.mahmoud@kasralainy.edu.eg

${ }^{1}$ Department of diagnostic and interventional radiology, Cairo University, Giza, Egypt

Full list of author information is available at the end of the article
}

Approximately $80-90 \%$ of cases of MS are relapsingremitting in nature. The remaining $10-20 \%$ has a primary progressive course with gradually worsening symptoms. Depression in MS patients can compromise cognitive functions, lead to suicide attempts, impair relationships and reduce compliance with disease-modifying treatments $[2,3]$, with reported rates of missed diagnosis of approximately $23-30 \%$ and rates of inadequate treatment of approximately $20-36 \%$ of those diagnosed with depression [4].

A number of reasons may explain the association of MS with depression including the psychosocial effects of 
MS disability, the direct effect of lesions on brain structures that are involved in regulating and maintaining mood state and the untoward effects of disease-modifying drugs (DMD) for treating MS, which may be associated with mood changes and immune dysfunction [5]. The observation that depressive symptoms can precede the onset of neurological symptoms suggests that depression may be related to early disease-specific processes [6]. Specific MS pathologies may trigger the onset of depression. In major depressive disorder (MDD), there are several brain abnormalities that have been found, such as frontal and limbic atrophy and decreased FA of the fronto-limbic white matter tracts [7].

The clinical presentation of depression in patients with MS is very similar to that of patients with major depressive disorder (MDD) without MS [8].

The human limbic system consists of a group of interconnected nuclei and cortical structures that mediate behaviour, memory and emotions. The limbic system is implicated in the pathology of a wide spectrum of psychiatric disorders [9]. The Papez circuit involves bidirectional interaction between the anterior thalamic nuclei and hippocampus via the cingulum bundle and fornix. The Papez circuit is critical to recall and memory encoding [10]. The uncinate fasciculus (UF) connects the prefrontal cortex to the anterior temporal pole, completing the thalamic-hippocampal-prefrontal circuit [2]. Previous studies have shown MS-related damage in the fornix [11], cingulum [12] and UF [13].

Additionally, compared to patients without depression and with MS (nDMS), patients with depression and MS (DMS) exhibit more severe atrophy of the frontal cortex [14] and damage to the frontal white matter, which is detected as decreased FA [15].

The diffusion tensor imaging (DTI) is considered one of the recent non-invasive functional MR techniques for studying brain tissue microstructure. The FA (fractional anisotropy) detects the directionality of diffusion of water molecules inside the tissue. White matter abnormalities that cause loss of axonal organisation produce lower FA values [16].

DTI has a higher specificity than conventional MRI in the assessment of MS due to the variable heterogeneous pathological changes of the disease. It can be used to grade MS injury in the white matter lesions, the normalappearing white matter, the normal-appearing grey matter, the optic nerves and the spinal cord as decreased FA and increased mean diffusivity may precede the appearance of MS lesions on conventional images [17].

To our knowledge, imaging studies investigating the changes in the limbic system in DMS patients are lacking. Therefore, in this study, we aimed to investigate the microstructural changes in the white matter tracts of the limbic system using the DTI technique in patients with MS who presented with depressive symptoms.

\section{Methods}

Forty patients were included in this prospective study between November 2016 and October 2017. Patients' ages ranged between 21 and 36 years with a mean age of 27 . Thirty patients with MS (24 females and 6 males) were divided into two groups. Group 1 included 20 patients with relapsing-remitting MS (RRMS) with depressive symptoms diagnosed according to McDonald's criteria 2010 and diagnosed with depression according to DSM-V (Diagnostic and Statistical Manual of Mental Disorders). Group 2 consisted of 10 patients with MS without symptoms of depression. Both depressed and non-depressed groups were similar with regard to several variables, including demographics, cognitive performance, disease duration and lesion load. The control group consisted of 10 age-matched healthy individuals. Written informed consent was signed by all patients before the examination. The study was conducted with the approval of our institutional review board.

Inclusion criteria

- RRMS with Expanded Disability Status Scale (EDSS) $\leq 5$

- Clinically detected depression according to DSM-V (with BDI "Beck Depression Inventory" ranging from 9 to 29$)$

\section{Exclusion criteria}

- Severe or extreme depression $(\mathrm{BDI}>30)$ requiring hospitalisation or acute interventions

- Contraindications to MRI

- Receiving antidepressant drugs

All patients were subjected to the following:

- Full neurological and clinical examination by an experienced neurologist

- Beck Depression Inventory (BDI): The BDI was developed in 1961, adapted in 1969, and copyrighted in 1979. It becomes one of the most widely used measures to assess the severity of depression whether in adolescents or adults. The BDI is composed of 21 questions or items with four possible responses. Each response is assigned to a score from zero to three, with the sum of the scores indicating the severity of the symptom with higher scores indicating a more severe depression [18]. For people who have been clinically diagnosed, scores from 0 to 9 represent minimal depressive symptoms, scores from 10 to 16 indicate mild depression, 
scores from 17 to 29 indicate moderate depression and scores from 30 to 63 indicate severe depression

- Tests for cognitive skills (PASAT): Paced Auditory Serial Addition Test (PASAT) (B). The PASAT is a measure of cognitive function that specifically assesses auditory information processing speed and flexibility, as well as calculation ability [19]. This assessment was initially developed by Gronwall in 1977. Stimulus presentation rates were adapted for use with patients with MS by Rao and colleagues in 1989

\section{MRI examination}

MRI was performed without prior preparation or anaesthesia. Imaging was performed using a standard 1.5 Tesla unit (Achieva, Philips). A standard head coil was used. The sequences obtained were axial T1 (TR $450 \mathrm{~ms}$, TE $15 \mathrm{~ms}$, flip angle $69^{\circ}$, matrix $180 \times 169$, FOV $210 \times 236$, number of excitation: 2, slice thickness: 6.0/1.5), axial T2 (TR $3619 \mathrm{ms,}$ TE $100 \mathrm{~ms}$, flip angle $90^{\circ}$, matrix $192 \times 165$, FOV $210 \times 236$, number of excitation: 2, slice thickness: 6.0/1.5), axial and sagittal FLAIR (TR $2000 \mathrm{~ms}$, TE $120 \mathrm{~ms}$, flip angle 90', matrix $208 \times 192$, FOV $210 \times 236$, number of excitation: 2 , slice thickness: 6.0/1.5) and diffusion WIs (diffusion weighting factor of zero, 500 and $1000 \mathrm{~s} / \mathrm{mm} 2$, TR $4100 \mathrm{~ms}$, TE $115 \mathrm{~ms}$, flip angle $90^{\circ}$, matrix $132 \times 105$, FOV $210 \times 236$, number of excitation: 2 , slice thickness: 6.0/1.5).

DTI obtained for all patients consisted of a single-shot, spin-echo echoplanar sequence in 12 encoding directions. A diffusion weighting factor of $800 \mathrm{~s} / \mathrm{mm}^{2}$ was used. The imaging parameters were as follows: TR 8000 $\mathrm{ms}$, TE $67 \mathrm{~ms}$, flip angle $90^{\circ}$, matrix $112 \times 110$, FOV 210 $\mathrm{mm}$, number of excitations 2 , and slice thickness $2 \mathrm{~mm}$.

All images were transferred to a workstation (Philips Extended MR Workspace, 2.6.3.5) for post-processing.
FA maps, directionally encoded colour FA maps and 3D fibre tractography, were obtained.

FA values were measured in the main white matter connections of the limbic system, including the cingulum cingulate part (Fig. 1), UF (Fig. 2), fornix (Fig. 3) and anterior thalamic radiations (Fig. 4). Freehand drawings of the regions of interest (ROIs) were made at the FA colour map overlaid on T2 or FLAIR images. Measurements were performed at the normal-appearing white matter without demyelinating plaques. The number of voxels for each ROI ranged between 2 and 6. The multi-ROI technique was used, and a software algorithm tracked the white matter tracts that passed through these ROIs. Four pathways were identified bilaterally using a prior anatomical knowledge and established tractography protocols $[20,21]$.

Correlations were performed between the mean FA values of the white matter tracts in the depressed and non-depressed MS groups, between the depressed group and the normal healthy individuals and between the non-depressed group and normal healthy group.

\section{Statistical analysis}

The results are expressed as the mean \pm standard deviation or number (percentage). Correlations between the FA of the different groups were performed using the paired Student's $t$ test. $P$ values below 0.05 were considered statistically significant. SPSS software (version 16 windows) was used for data analysis.

\section{Results}

The mean FA values of the four examined white matter tracts in the different study groups are discussed in detail in Table 1.

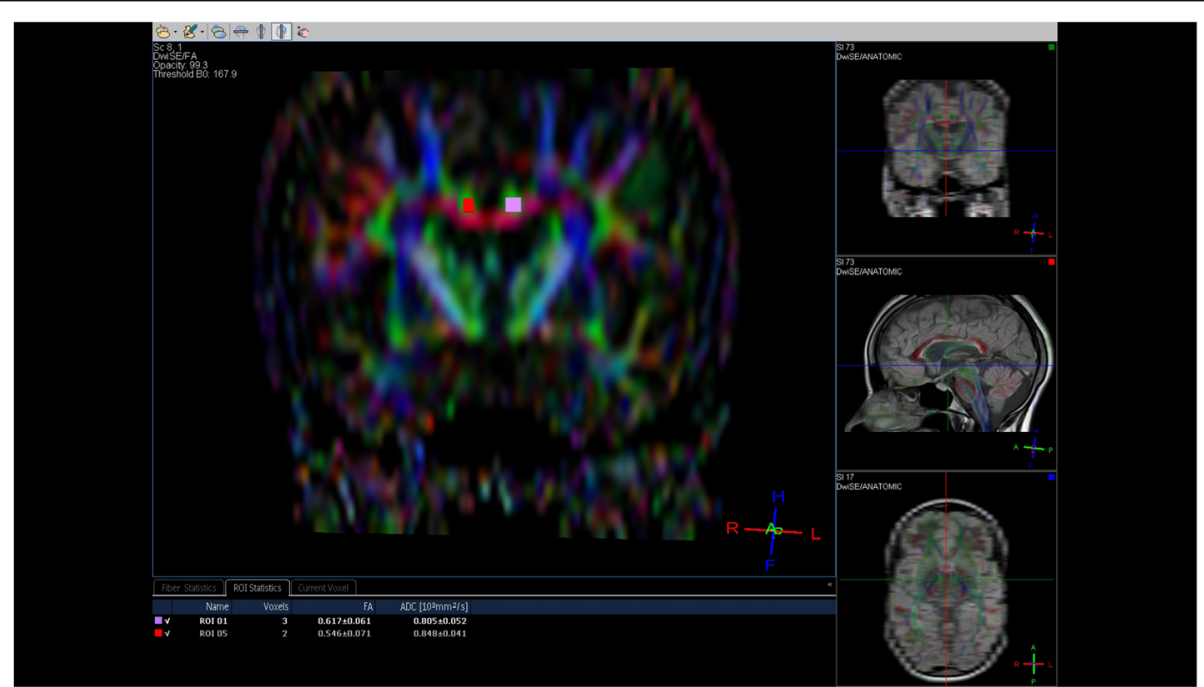

Fig. 1 32-year-old female patient known to have MS with recent onset of depressive symptoms (BDI 15). Colour-coded FA maps of the cingulum cingulate showing FA reduction of the right and left cingulum (FA $0.617 \pm 0.061$ on the right and $0.546 \pm 0.071$ on the left) 


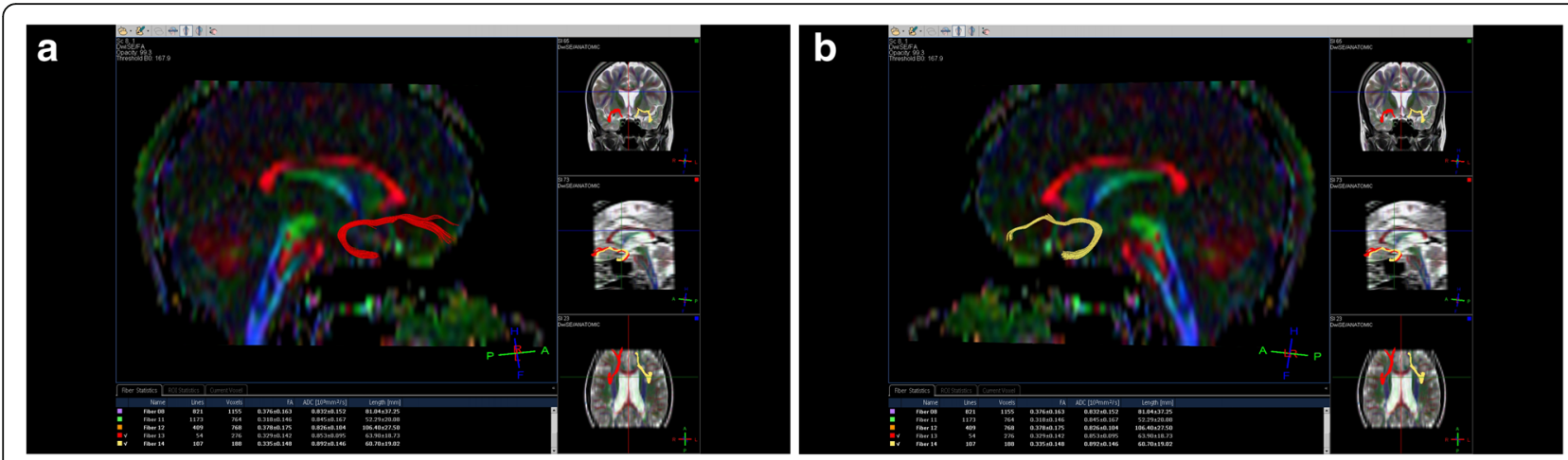

Fig. 2 26-year-old male patient known to have MS and presented with depression (BDI 18). Colour-coded FA maps with 3D fibre tractography of the uncinate fasiculi (UF). $\mathbf{a}$ Right side and $\mathbf{b}$ left side showing FA reduction of the right and left tracts (FA $0.329 \pm 0.142$ on the right and $0.335 \pm$ 0.148 on the left)

Compared with nDMS patients, DMS patients showed decreased FA values with significant differences in the right and left cingulum $(P<0.0111$ on the right and $P<$ 0.0142 on the left), right and left UF $(P<0.0001$ on the right and $P<0.0076$ on the left) and the right and left fornix ( $P<0.0001$ on both sides). By contrast, no significant differences were found in the mean FA values of the anterior thalamic radiations between both groups $(P<0.3264$ on the right and $P<0.1622$ on the left). A comparison of the mean FA values of the white matter tracts on both sides in the same patient revealed no significant differences between the right and left tracts.

A comparison of the mean FA values of the four white matter tracts between nDMS patients and age-matched normal healthy individuals revealed a significant reduction in the mean FA value of the left cingulum only $(P<$ $0.0054)$. No significant difference was detected in the other examined tracts.

By comparing the mean FA values of the four white matter tracts between DMS patients and age-matched normal healthy individuals, a significant reduction in the mean FA value of the right and left cingulum $(P<0.0001$ on the right and $P<0.0004$ on the left), UF $(P<0.0001$ on the right and $P<0.0168$ on the left) and the fornix $(P<0.0001$ on the right and $P<0.001$ on the left) was found. No significant difference was detected in the ATR between the two groups.

\section{Discussion}

In this study, we investigated microstructural changes in the white matter connections of the fronto-limbic system in patients with depression and MS. The white matter changes were reflected as reduced FA values using the DTI technique. Decreased FA values were found in the cingulum (cingulate part), fornix and UF in patients with DMS compared to those in nDMS patients.

The UF connects the temporal lobe, which contains the hippocampus and amygdala, with the prefrontal cortex, and is considered to be involved in cognitive functions [22]. Spatial memory deficits in depressed and cognitively impaired MS patients are linked to lesions in the UF [12]. The UF was also important for episodic verbal memory function on the California Verbal Learning

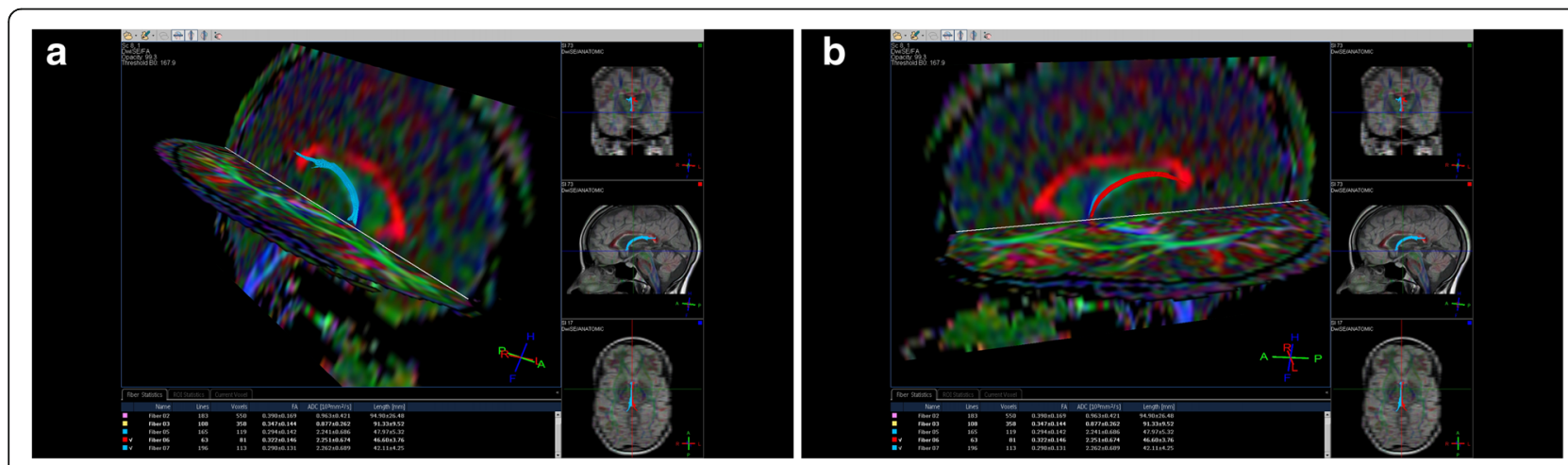

Fig. 3 30-year-old female patient known to have MS with depressive symptoms (BDI 25). Colour-coded FA maps overlaid on FLAIR images with 3D fibre tractography of the fornix. a Right side and $\mathbf{b}$ left side showing FA reduction of the right and left fornix (FA $0.290 \pm 0.131$ on the right and $0.322 \pm 0.146$ on the left) 


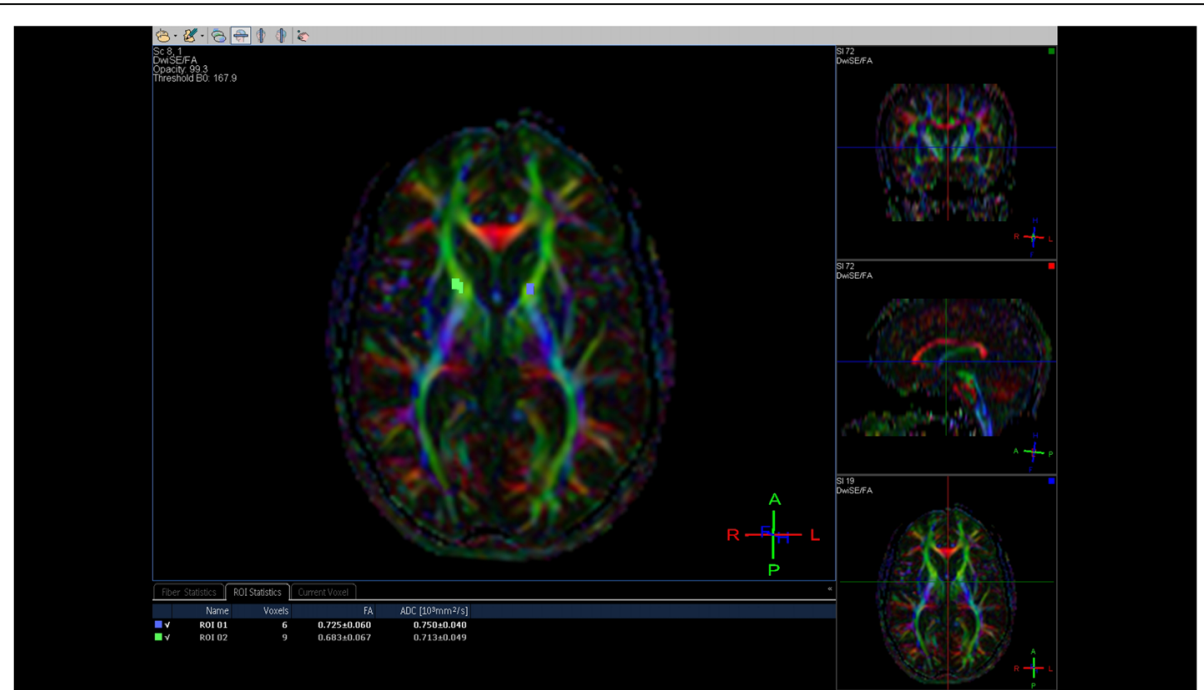

Fig. 4 29-year-old female patient known to have MS and recently presented with depression (BDI 21). Colour-coded FA maps and 3D fibre tractography of the anterior thalamic radiations showing no significant FA reduction of the right and left tracts

Test (CVLT) across a cohort of patients with MS and controls [23].

The cingulum is associated with attention/executive function and has been implicated in other realms of cognitive performance in patients with depression and MS. Moreover, there are associations between the FA values of the cingulum and the PASAT, Benton Visual Retention Test (BVRT) and CVLT-II [24].

The fornix is considered the primary hippocampal efferent. The fornix has been associated with differences in MS on verbal memory tests, including CVLT-II [23, 24] and BVRT [25]. A link between the FA of the fornix and verbal memory performance was identified in a previous study [26], but this relationship was not independent of thalamic volume. Additionally, the thalamic volume and FA of the fornix were strongly associated, which has been previously shown [25].
The damage induced by MS in the hippocampal-thalamic-prefrontal network and its contributions to depression and specific cognitive domains was assessed by Kern et al. [26]. These authors found that compared to healthy controls, patients with RRMS had a reduced mean FA of the UF, fornix and cingulum which agreed with our findings. Moreover, compared with controls, patients with RRMS exhibited reduced thalamic and hippocampal volumes.

In another study performed by van Geest et al. [7] on 22 patients with depression and MS, 21 nDMS patients and 12 healthy controls, DMS patients had lower FA than did nDMS patients in the left UF $(P=0.05)$. These authors also found that DMS patients had lower FA in the left cingulum $(P=0.04)$ and left UF $(P=0.03)$ than did healthy controls. By contrast, no differences were found between nDMS patients and healthy controls. Also, these results are matched with our findings.

Table 1 Illustrates the mean FA values of the examined white matter tracts in different study groups and statistical results

\begin{tabular}{|c|c|c|c|c|c|c|}
\hline & \multicolumn{3}{|c|}{ Mean FA value $( \pm S D)$} & \multirow{2}{*}{$\begin{array}{l}\text { DMS } \\
\text { vs nDMS, } P \text { value }\end{array}$} & \multirow{2}{*}{$\begin{array}{l}\text { nDMS } \\
\text { vs } H C, P \text { value }\end{array}$} & \multirow{2}{*}{$\begin{array}{l}\text { DMS } \\
\text { vs HC, } P \text { value }\end{array}$} \\
\hline & $\overline{D M S}(n=20)$ & nDMS $(n=10)$ & $\mathrm{HC}(n=10)$ & & & \\
\hline Right cingulum & $0.5690(0.0529)$ & $0.6220(0.0444)$ & $0.6690(0.0431)$ & 0.0111 & 0.0273 & 0.0001 \\
\hline Left cingulum & $0.5660(0.0811)$ & $0.6350(0.0232)$ & $0.6740(0.0313)$ & 0.0142 & 0.0054 & 0.0004 \\
\hline Right uncinate fasciculus & $0.4035(0.0673)$ & $0.5250(0.0425)$ & $0.5300(0.0450)$ & 0.0001 & 0.8012 & 0.0001 \\
\hline Left uncinate fasciculus & $0.4485(0.0825)$ & $0.5310(0.0522)$ & $0.5220(0.0543)$ & 0.0076 & 0.7099 & 0.0168 \\
\hline Right fornix & $0.3820(0.0711)$ & $0.5070(0.0419)$ & $0.5090(0.0398)$ & 0.0001 & 0.9141 & 0.0001 \\
\hline Left fornix & $0.3435(0.0587)$ & $0.4610(0.0536)$ & $0.4770(0.0343)$ & 0.0001 & 0.4373 & 0.0001 \\
\hline Right anterior thalamic radiation & $0.5815(0.0939)$ & $0.6120(0.0266)$ & $0.6230(0.0445)$ & 0.3264 & 0.5106 & 0.1985 \\
\hline Left anterior thalamic radiation & $0.5775(0.1057)$ & $0.6290(0.0557)$ & $0.6150(0.0395)$ & 0.1622 & 0.5248 & 0.2907 \\
\hline
\end{tabular}


Shen et al. [27] studied white matter and grey matter abnormalities in 15 patients with RRMS with depressive symptoms compared to those in 15 age- and gendermatched normal controls. Areas of reduced FA were detected in the grey matter and white matter, such as the frontal lobe and particularly in the precentral gyrus, limbic system, cingulate gyrus, temporal lobe, occipital lobe and parietal lobe.

Our results could have an impact on the management of MS patients with depressive manifestations. Follow-up of the patients with MRI and diffusion tensor imaging after different therapeutic procedures can detect the improvement in the FA values of the affected white matter tracts and thus can aid in the detection of patients' improvement, side by side with clinical assessment. Further studies are recommended to assess the prognostic value of DTI in DMS patients.

This study has several strengths, but it also has some weaknesses that prompt future investigations. For instance, the sample size is relatively small. Another limitation is that we did not include a functional assessment of the cortical structures involved in the pathology of depression in patients with MS. Instead, we focused only on the white matter tract assessment using DTI. Moreover, we studied only the white matter changes in the fronto-limbic pathway. Thus, we likely omitted contributions from other affected structures in MS that are related to cognitive dysfunction, such as the corpus callosum, basal ganglia and neocortex. We recommend further studies with a larger number of cases, as well as functional and volumetric assessments of the fronto-limbic pathway.

\section{Conclusion}

Patients with depression and MS showed more pronounced microstructural damage in the major white matter connections of the limbic pathway, namely, the UF, cingulum and fornix. These changes can be detected by DTI as decreased FA values in DMS patients compared to those in nDMS patients.

\footnotetext{
Abbreviations

BDI: Beck Depression Inventory; BVRT: Benton Visual Retention Test; DMS: Depressed MS patients; DSM: Diagnostic and Statistical Manual of Mental Disorders; DTI: Diffusion tensor imaging; EDSS: Expanded Disability Status Scale; FA: Fractional anisotropy; MDD: Major depressive disorder; MS: Multiple sclerosis; nDMS: Non-depressed MS patients; PASAT: Paced Auditory Serial Addition Test; RRMS: Relapsing-remitting MS; UF: Uncinate fasciculus
}

\section{Acknowledgements}

Not applicable.

\section{Authors' contributions}

TAH conceived of the study and participated in its design and coordination. SFE participated in the design of the study and performed the DTI analysis and FA measurements. BEM is the editor of the manuscript, helped in the FA measurements and performed the statistical analysis. ME patients collection and clinical correlation. All authors read and approved the final manuscript.
Funding

Not applicable (no funding received for the study)

\section{Availability of data and materials}

The datasets used and analysed during the current study are available from the corresponding author on reasonable request.

\section{Ethics approval and consent to participate}

The study protocol was approved by the research committee of the Faculty of Medicine, Cairo University, Egypt, on 19 July 2016 followed by the final approval of the university committee on 25 July 2016. Written informed consent was signed by all patients participated in the study before the MRI examination.

\section{Consent for publication}

All patients included in this research were fully conscious and older than 16 years old and gave written informed consent to publish the data contained within this study.

\section{Competing interests}

The authors declare that they have no competing interests.

\section{Author details}

${ }^{1}$ Department of diagnostic and interventional radiology, Cairo University, Giza, Egypt. ${ }^{2}$ Department of neurology, Agouza Hospital, Cairo, Egypt.

Received: 31 May 2019 Accepted: 19 August 2019

Published online: 17 September 2019

\section{References}

1. Sommerlad A., Price G. and Trip A. Management of neuropsychiatric symptoms in multiple sclerosis. Progress in Neurology and Psychiatry 2014; March/April 14:17

2. Feinstein A (2011) Multiple sclerosis and depression. Mult Scler J 17(11): 1276-1281

3. D'Alisa S, Miscio G, Baudo S et al (2006) Depression is the main determinant of quality of life in multiple sclerosis: a classification-regression (CART) study. Disabil Rehabil 28:307-314

4. Marrie RA, Horwitz R, Cutter G (2009) The burden of mental comorbidity in multiple sclerosis: frequent, under diagnosed, and under treated. Mult Scler. 15(3):385-392

5. Wallin MT, Wilken JA, Turner AP et al (2006) Depression and multiple sclerosis: Review of a lethal combination. J Rehabil Res Dev. 43(1):45-62

6. Vattakatuchery JJ, Rickards H, Cavanna AE (2011) Pathogenic mechanisms of depression in multiple sclerosis. J Neuropsychiatry Clin Neurosci. 23(3):261-276

7. van Geest Q, Boeschoten RE, Keijzer MJ et al (2019) Fronto-limbic disconnection in patients with multiple sclerosis and depression. Mult Scler. 25(5):715-726. https://doi.org/10.1177/1352458518767051

8. Hasselmann H, Bellmann-Strobl J, Ricken R et al (2016) Characterizing the phenotype of multiple sclerosis associated depression in comparison with idiopathic major depression. Mult Scler 22(11):1476-1484

9. Patestas MA, Gartner LP (2006) "Limbic system," in A Textbook of Neuroanatomy, vol 344. Blackwell, Oxford, England, p 359

10. Aggleton JP, Sahgal A (1993) The contribution of the anterior thalamic nuclei to anterograde amnesia. Neuropsychologia 31(10):1001-1019

11. Kern KC, Ekstrom AD, Suthana NA et al (2012) Fornix damage limits verbal memory functional compensation in multiple sclerosis. Neuro Image 59(3): 2932-2940

12. Mesaros S, Rocca MA, Kacar K et al (2012) Diffusion tensor MRI tractography and cognitive impairment in multiple sclerosis. Neurology 78(13):969-975

13. Fink F, Eling P, Rischkau E et al (2010) The association between California verbal learning test performance and fibre impairment in multiple sclerosis: evidence from diffusion tensor imaging. Mult Scler. 16(3):332-341

14. Nunnari D, De Cola MC, D'Aleo G et al (2015) Impact of depression, fatigue, and global measure of cortical volume on cognitive impairment in multiple sclerosis. Biomed Res Int 2015:519785

15. Feinstein A, O'Connor P, Akbar N et al (2010) Diffusion tensor imaging abnormalities in depressed multiple sclerosis patients. Mult Scler 16(2):189-196 
16. Razek AAKA, El-Serougy L, Abdelsalam M et al (2018) Differentiation of residual/recurrent gliomas from postradiation necrosis with arterial spin labeling and diffusion tensor magnetic resonance imaging-derived metrics. Neuroradiology 60:169-177

17. Filippi M, Pagani E, Preziosa P, Rocca MA (2016) The role of DTI in multiple sclerosis and other demyelinating conditions. In: Van Hecke W, Emsell L, Sunaert S (eds) Diffusion Tensor Imaging. Springer, New York, NY

18. Beck A, Steer R, Brown G (1996) Beck Depression Inventory, 2nd edn. Psychological Corporation, San Antonio, TX, E.U. [Google Scholar]

19. Tombaugh TN (2006) A comprehensive review of the Paced Auditory Serial Addition Test (PASAT). Arch Clin Neuropsychol 21:53-76. https://doi.org/10.1 016/j.acn.2005.07.006

20. Concha L, Beaulieu C, Gross DW (2005) Bilateral limbic diffusion abnormalities in unilateral temporal lobe epilepsy. Ann Neurol 57:188-196

21. Wakana S, Caprihan A, Panzenboeck MM et al (2007) Reproducibility of quantitative tractography methods applied to cerebral white matter. Neurolmage 36(3):630-644

22. Von Der Heide RJ, Skipper LM, Klobusicky E et al (2013) Dissecting the uncinate fasciculus: disorders, controversies and a hypothesis. Brain 136(Pt 6):1692-1707

23. Fink F, Eling P, Rischkau E (2010) Bet al. The association between California verbal learning test performance and fiber impairment in multiple sclerosis: evidence from diffusion tensor imaging. Mult Scler. 16(3):332-341

24. Dineen RA, Vilisaar J, Hlinka J et al (2009) Disconnection as a mechanism for cognitive dysfunction in multiple sclerosis. Brain 132(1):239-249

25. Dineen RA, Bradshaw CM (2012) Constantinescu, et al. Extra-hippocampal subcortical limbic involvement predicts episodic recall performance in multiple sclerosis. PLOS One 7(10):e44942

26. Kern KC, Gold SM, Lee B et al (2015) Thalamic-hippocampalprefrontal disruption in relapsing-remitting multiple sclerosis. Neurolmage Clin 8:440-447

27. Shen Y, Bai L, Gao Y et al (2014) Depressive symptoms in multiple sclerosis from an in vivo study with TBSS. Biomed Res Int 2014:148465

\section{Publisher's Note}

Springer Nature remains neutral with regard to jurisdictional claims in published maps and institutional affiliations.

\section{Submit your manuscript to a SpringerOpen ${ }^{\circ}$ journal and benefit from:}

- Convenient online submission

- Rigorous peer review

- Open access: articles freely available online

- High visibility within the field

- Retaining the copyright to your article

Submit your next manuscript at $\boldsymbol{\nabla}$ springeropen.com 